ARTÍCULOS ORIGINALES Rev Chil Salud Pública 2018, Vol 22(2): 161-170
JUAN PÉREZ

- FRANCO

Superintendencia de Seguridad Social; Departamento de Psiquiatría y Salud Mental Sur, Universidad de Chile.

jperez@suseso.cl

MACARENA CANDIA Periodista Superintendencia de Seguridad Social Articulo recibido el 02/04/2018 Aceptado el 08/08/2018

\section{SUCIO Y DESPRECIADO. RIESGO PSICOSOCIAL EN TRABAJADORAS DE CASA PARTICULAR EN CHILE (*)}

\author{
Dirty and demeaning. Psychosocial risk in female \\ DOMESTIC WORKERS IN CHILE
}

\section{RESUMEN}

Introducción: El trabajo doméstico remunerado se encuentra socialmente muy desvalorado y se estima que es una actividad con alto riesgo psicosocial laboral (RPSL).

Objetivo: Verificar el nivel de RPSL y su asociación con variables de salud, sociodemográficas y de empleo en trabajadoras de casa particular (TCP) en Chile.

Material y método: Estudio transversal cuantitativo y cualitativo, con una muestra noprobabilística. Se aplicó el cuestionario SUSESO/ISTAS21 (COPSOQ 1) a través de dirigentes de TCP en todo Chile. Se realizó análisis estadístico. Posteriores sesiones de discusión con las mismas dirigentes, fueron registradas, transcritas y analizadas.

Resultados: Se aplicó un total de 291 cuestionarios. Riesgo alto en exigencias emocionales, esconder emociones, posibilidades de desarrollo, relación con los superiores. Riesgo bajo en exigencias cuantitativas, sensoriales, conflicto de rol, inseguridad del contrato, influencia, sentido del trabajo. Escalas de salud bajo la media chilena (peor salud) no se asociaron con variables sociodemográficas, ni con horas semanales trabajadas, ni con actividades de cuidado. Hubo correlaciones más bien pequeñas entre salud y RPSL, salvo para exigencias emocionales, integración en la empresa y estima. Dirigentes sugieren que pobre salud mental se debe no tanto a un elevado RPSL, sino que a la baja autoestima por devaluación del propio trabajo y vergüenza.

Discusión: TCP son sorprendentemente autónomas en su trabajo, pero tienen un alto riesgo en exigencias emocionales y una mala salud; tienen una actitud ambivalente ante sus empleadoras, dado el abuso por largas jornadas de trabajo pero también otorgan alta valoración al liderazgo. Principal problema es la baja autoestima expresada como vergüenza por un trabajo socialmente desvalorado más que el riesgo psicosocial.

Palabras clave: riesgo psicosocial; salud ocupacional; trabajo doméstico femenino.

Financiamiento: Este estudio no contó con financiamiento.

Conflicto de interés: Los autores declaran no tener conflictos de interés.

(*) Presentado inicialmente en 6th COPSOQ International Workshop, Santiago de Chile, 8-10 noviembre, 2017. 


\section{ABSTRACT}

Background: Paid domestic work is socially undervalued and it is estimated to be an activity with high psychosocial risk (PSR).

Objective: To verify the level of PSR and its association with health, sociodemographic and employment variables in female domestic workers (FDW) in Chile.

Material and method: Quantitative and qualitative cross-sectional study whit a non-probabilistic sample. The SUSESO / ISTAS21 questionnaire (COPSOQ 1) was administred to FDW leaders throughout Chile. Statistic analysis was carried out. Subsequent discussion sessions with the same leaders were recorded, transcribed and analyzed.

Results: A total of 291 FDW participated in the study. High risk was detected in emotional demands, hiding emotions, possibilities of development, relationship with superiors, and low risk in quantitative, and sensory demands, role conflict, contract insecurity, influence, and sense of work. Health scores below the Chilean average (worse health) were not associated with sociodemographic variables, nor with weekly hours worked, nor with care activities. There were rather small correlations between health and PSR, except for emotional demands, integration in the company and esteem. FDW leaders suggest that poor mental health is due not so much to a high PSR, but to low self-esteem due to devaluation of one's work and shame.

Discussion: FDW are surprisingly autonomous in their work, but have high risk in emotional demands and ambivalent attitudes towards their employers, given the abuse of long working hours. They also highly value leadership. The main problem is low self-esteem expressed as shame due to socially devalued work, and not so much psychosocial risk.

Keywords: psychosocial risk; occupational health; female domestic work

\section{INTRODUCCIÓN}

El trabajo doméstico remunerado en muchos países de Latinoamérica y el Caribe constituye la principal fuente de trabajo femenino no agrícola. ${ }^{1}$ Las trabajadoras de casa particular (TCP) son mayoritariamente mujeres, sus condiciones de trabajo están entre las más precarias y sufren serios déficit de trabajo decente. ${ }^{2} \mathrm{~A}$ las TCP se les delega el trabajo "espiritualmente degradante" ${ }^{3}$, un trabajo muy desvalorado porque reemplaza al trabajo doméstico femenino no remunerado que ya resultaba invisible ${ }^{3}$, ha sido tradicionalmente ignorado en legislaciones y se encuentra mucho más desamparado socialmente cuando se lo compara con otros trabajos. ${ }^{2}$ Probablemente contribuye a su invisibilidad el que se realice precisamente en un ámbito doméstico, que por un lado es de difícil fiscalización, pero por otro es un "telón inadvertido en la vida de las personas ${ }^{\prime 2, \text { p.13. }}$. Las TCP suelen trabajar más horas de las permitidas legalmente, y por lo general son peor remuneradas que en otras actividades.
La frecuente situación de gran diferencia social y cultural entre los empleadores y las TCP, las hace particularmente vulnerables al abuso o la negociación, manteniendo relaciones afectivas con la familia que las emplea llevándolas a tomar decisiones que no las benefician. ${ }^{1}$ De estas trabajadoras no solo se espera la venta de su fuerza de trabajo para limpiar, lavar, planchar o cocinar, sino también la distribución de afectos, es decir, que desarrolle sus labores con esmero y cariño. ${ }^{4}$

Para las trabajadoras extranjeras, debe agregarse la dificultad de adaptarse a un entorno cultural diferente y la falta de redes sociales. ${ }^{4,8}$ La gran incorporación de mujeres migrantes a este trabajo ha sido una marcada característica contemporánea. La encuesta CASEN 2015 muestra que 1 de cada 4 mujeres inmigrantes está trabajando en el servicio doméstico, de modo que para ellas esta actividad es la principal fuente de trabajo. ${ }^{9}$

A pesar de que no han faltado estudios que revelen las características sociodemográficas 
y de empleo de estas trabajadoras, ha existido menos preocupación por el riesgo psicosocial en esta actividad $^{10,11}$, y por el impacto de este riesgo sobre la salud mental de estas trabajadoras. ${ }^{12}$

Este estudio, de carácter transversal, exploratorio y descriptivo, cuantitativo y cualitativo, tuvo como objetivo mostrar el comportamiento de las variables de riesgo psicosocial laboral (RPSL) en los hogares considerados como lugar de trabajo. Nuestra hipótesis principal fue que los hogares en los que se desempeñan estas trabajadoras debían mostrar altos índices de RPSL, lo que debería reflejarse en las escalas de salud mental de las trabajadoras y estar asociado a actividades de cuidado. El trabajo cualitativo tuvo el objetivo de explorar las hipótesis que se dieran las propias trabajadoras acerca de los resultados observados.

\section{MATERIAL Y MÉTODO}

Se utilizó el cuestionario autoaplicado SUSESO/ ISTAS21 (COPSOQ 1) 13,14 para medición de RPSL, validado y estandarizado en Chile, que permite calificar la exposición de cada trabajador a niveles alto, medio y bajo de RPSL en 18 subdimensiones o escalas. ${ }^{15}$ Con estos datos se calculó la prevalencia de cada uno de los tres niveles de riesgo por subdimensión considerando a todos los hogares como si fuesen un solo lugar de trabajo. ${ }^{15,16}$ También se analizó la frecuencia de respuestas con puntajes extremos de riesgo en cada escala.

El cuestionario posee escalas de salud general (SG), salud mental (SM) y vitalidad (VT), tomadas del cuestionario SF-36, también validado y estandarizado en Chile. ${ }^{17}$ Estas escalas mostraron un comportamiento normal y se compararon con las medias del SF-36 para Chile ${ }^{17}$ mediante una prueba t de Student. Además se dicotomizó la muestra entre ser "caso" de salud (tener dos o más desviaciones estándar por debajo de la media chilena) y "control", y así se calcularon algunos OR, por ejemplo, con haber sufrido algún tipo de acoso.

Los cálculos se realizaron con la aplicación SPSS.

Los autores tomaron contacto durante 2015 con dirigentes sindicales de las TCP con las que se discutió y precisó diversas características de su trabajo, se revisó con ellas el cuestionario, se eliminaron escalas que no eran adecuadas, y se agregaron preguntas que precisaban las condiciones sociales y laborales de las TCP.

Las dirigentes sugirieron además cuatro condiciones que, en su opinión, generarían una alta carga emocional: recibir órdenes de dos o más personas, cuidar niños menores de 12 años, cuidar ancianos o enfermos y cuidar mascotas, con las que se construyó una escala de riesgo doméstico.

Durante ese mismo año los autores participaron en encuentros nacionales de TCP donde se aplicaron los cuestionarios, asegurándonos de la comprensión lectora en personas que suponíamos de baja escolaridad. En algunas regiones fueron las propias dirigentes quienes aplicaron los cuestionarios siguiendo similares instrucciones de resolver dudas y estar disponibles en caso de problemas de comprensión lectora. La muestra seleccionada fue no probabilística y por conveniencia y no tiene un carácter representativo de las TCP en Chile.

Se dio especial relevancia al resguardo del anonimato y la confidencialidad, lo que fue discutido tanto con las dirigentes como en los encuentros que tuvo el equipo con las trabajadoras.

Una vez recogidos, digitalizados, tabulados y analizados los cuestionarios se tuvo sesiones de discusión de los resultados con las dirigentes las que fueron registradas en audio, transcritas y analizadas. En esas sesiones se discutieron los motivos por los que aparecieron los resultados observados y se buscaron hipótesis explicativas, siempre junto a las dirigentes. El análisis final, sin embargo, es solo de nuestro equipo.

El diseño y ejecución del trabajo contempló los criterios éticos establecidos por ley para las instituciones públicas en Chile, cumpliendo altos estándares de probidad y transparencia.

\section{RESULTADOS}

Entre septiembre y octubre de 2015 se recogieron 291 cuestionarios suficientemente completos para el análisis.

De las 291 TCP incluidas en la muestra, 235 
$(81,0 \%)$ fueron chilenas y $55(19,0 \%)$ fueron extranjeras (1,4\% hombres). Un $14,5 \%$ de las extranjeras tenía edades menores a 36 años; entre las chilenas solo un $3 \%$ estuvo en esa categoría. Las chilenas tuvieron una media de 9,2 años de escolaridad y las extranjeras una media de 11,7 años ( $p<0,001)$.

Las TCP chilenas trabajan mayoritariamente $(70,4 \%)$ en modalidad "puertas afuera". Las extranjeras, en cambio, en su gran mayoría $(78,4 \%)$ trabajan "puertas adentro".

La mediana de horas semanales de trabajo en puertas afuera es de 45 horas, y la mediana en puertas adentro es de 60 horas. Un 35,5\% trabajan hasta 8 horas diarias, un $41,5 \%$ entre 9 y 12 horas diarias, y un $23,0 \%$ trabaja más de 12 horas diarias; 7 trabajadoras registraron 90 y 98 horas semanales. Las TCP puertas afuera ganan menos que las TCP puertas adentro considerando iguales horas trabajadas, sin diferencia por nacionalidad.

Un 3,5\% de las TCP han sufrido algún tipo de acoso sexual, y un $24,6 \%$ algún tipo de acoso laboral, sin diferencias entre chilenas y extranjeras.

\section{Salud}

Los puntajes de salud entre chilenas y extranjeras resultaron sin diferencias significativas (t de Student, $p>0$,05). En la Tabla 1 puede observarse que los puntajes de SG, SMy VT entre las TCP son más bajos que la media chilena, es decir, tienen peor salud (t de Student; $p<0,01$ ).

Tabla 1. Puntajes de salud (SF-36) entre TCP y media para Chile. T de Student.

\begin{tabular}{|l|c|c|c|}
\hline Escalas & $\begin{array}{c}\text { TCP } \\
\text { media }\end{array}$ & $\begin{array}{c}\text { Chile* }^{*} \\
\text { media }\end{array}$ & Diferencia \\
\hline Salud General (SG) & 60,2 & 70,8 & $-10,6$ \\
\hline Salud Mental (SM) & 60,5 & 70,5 & $-10,0$ \\
\hline Vitalidad (VT) & 56,3 & 66,3 & $-10,0$ \\
\hline
\end{tabular}

*: media para Chile (Olivares, 2005); $\mathrm{p}<0,0001$
No hubo diferencias de puntajes de salud asociadas a variables sociodemográficas, empleo ni riesgo doméstico.Tampoco encontramos alguna asociación entre horas semanales trabajadas y puntajes de salud. Sí encontramos correlaciones significativas (rho de Spearman) entre escalas de RPSL y de salud (Tabla 2).

Las correlaciones más importantes fueron entre exigencias emocionales/SM ( $r$ ho $=-0,38$ ), estima/VT ( $r$ ho $=-0,32$ ); exigencias emocionales/ VT (rho = -0,30); integración en la empresa/SM (rho = -0,20); integración en la empresa/VT (rho $=-0,30)$, todas significativas con $p<0,001$. No hubo correlaciones significativas entre horas semanales y puntajes de salud. Se registró una alta OR $(4,75)$ entre acoso sexual y ser caso de salud mental, aunque fue significativa en el límite de confianza ( $p=0,045)$. No hubo relación significativa de las otras escalas de salud con acoso (laboral o sexual).

Suponiendo que las horas de trabajo semanal pudieran ser un proxi de abuso en el trabajo, estudiamos la correlación entre cantidad de horas semanales trabajadas y puntajes de salud (Pearson) y no encontramos correlación.

\section{Riesgo psicosocial}

No encontramos diferencias significativas en los puntajes de riesgo psicosocial entre chilenas y extranjeras.

Cuatro subdimensiones (Tabla 3) sobrepasan el umbralde riesgo alto (más del $50 \%$ de prevalencia de riesgo alto), ${ }^{16}$ (EM-“Exigencias emocionales", EE-"Exigencia de esconder emociones", PD-"Posibilidades de desarrollo", RS-"Relación con los superiores"), y otras tres (DP-“Preocupación por obligaciones en el hogar", CT-"Control del tiempo", RL-"Claridad del rol", ET-“Estima") se acercan al umbral. 
Tabla 2. Correlación (Spearman) entre escalas de riesgo psicosocial y escalas de salud. Trabajadoras de casa particular. 2015.

\begin{tabular}{|l|l|l|l|}
\hline Escalas de RPSL & $\begin{array}{l}\text { Salud } \\
\text { general }\end{array}$ & $\begin{array}{l}\text { Salud } \\
\text { mental }\end{array}$ & Vitalidad \\
\hline CU - Exigencias cuantitativas & $-0,16^{*}$ & $-0,30^{* *}$ & $-0,25^{* *}$ \\
\hline CO - Exigencias cognitivas & 0,03 & $-0,07$ & $-0,04$ \\
\hline EM - Exigencias emocionales & $-0,13$ & $-0,38^{* *}$ & $-0,30^{* *}$ \\
\hline EE - Exigencia de esconder emociones & $-0,06$ & $-0,25^{* *}$ & $-0,14^{*}$ \\
\hline ES - Exigencias sensoriales & 0,07 & $-0,01$ & 0,01 \\
\hline IN - Influencia & $-0,11$ & $-0,25^{* *}$ & $-0,18^{*}$ \\
\hline CT - Control del tiempo & $-0,08$ & $-0,19^{* *}$ & $-0,17^{*}$ \\
\hline PD - Posibilidades desarrollo & $-0,09$ & $-0,12$ & $-0,16^{*}$ \\
\hline ST - Sentido del trabajo & $-0,08$ & $-0,18^{* *}$ & $-0,24^{* *}$ \\
\hline IE - Integración a la empresa & $-0,08$ & $-0,20^{* *}$ & $-0,30^{* *}$ \\
\hline RL - Claridad de rol & $-0,22^{* *}$ & $-0,26^{* *}$ & $-0,24^{* *}$ \\
\hline CR - Conflicto de rol & $-0,12$ & $-0,26^{* *}$ & $-0,29^{* *}$ \\
\hline CL - Calidad del liderazgo & 0,02 & $-0,18^{* *}$ & $-0,26^{* *}$ \\
\hline RS - Relación con los superiores & $-0,03$ & $-0,21^{* *}$ & $-0,25^{* *}$ \\
\hline ET - Estima & $-0,05$ & $-0,29^{* *}$ & $-0,32^{* *}$ \\
\hline IC - Inseguridad del contrato & $-0,14$ & $-0,27^{* *}$ & $-0,22^{* *}$ \\
\hline IT - Inseguridad de condiciones del trabajo & $-0,15^{*}$ & $-0,21$ & $-0,21$ \\
\hline DP - Preocupación por responsabilidades en propia casa & 0,03 & $-0,06$ & $-0,01$ \\
\hline
\end{tabular}

*: Spearman, $p<0,05$

** : Spearman, $\mathrm{p}<0,01$

Tabla 3. Prevalencia de niveles de riesgo para los hogares como un único lugar de trabajo. TCP, Chile, 2015.

\begin{tabular}{|l|c|c|c|}
\hline Subdimensiones & $\begin{array}{c}\text { Riesgo } \\
\text { alto \% }\end{array}$ & $\begin{array}{c}\text { Riesgo } \\
\text { medio \% }\end{array}$ & $\begin{array}{c}\text { Riesgo } \\
\text { bajo \% }\end{array}$ \\
\hline CU -Exigencias cuantitativas & 32,6 & 20,7 & 46,7 \\
\hline CO -Exigencias cognitivas & 15,6 & 18,0 & 66,4 \\
\hline EM -Exigencias emocionales & 54,8 & 23,8 & 21,5 \\
\hline EE -Ex esconder emociones & 58,9 & 18,3 & 22,8 \\
\hline ES -Exigencias sensoriales & 18,9 & 22,7 & 58,4 \\
\hline IN -Influencia & 35,7 & 14,5 & 49,8 \\
\hline CT -Control del tiempo & 45,9 & 31,0 & 23,1 \\
\hline PD -Posibilidades de desarrollo & 59,9 & 28,0 & 12,1 \\
\hline ST -Sentido del trabajo & 41,4 & 10,4 & 48,2 \\
\hline IE -Integración en la empresa & 37,7 & 39,3 & 23,0 \\
\hline RL -Claridad de rol & 47,0 & 18,2 & 34,7 \\
\hline CR -Conflicto de rol & 24,3 & 24,3 & 51,4 \\
\hline CL -Calidad del liderazgo & 37,4 & 26,8 & 35,8 \\
\hline RS -Relación con los superiores & 54,4 & 19,2 & 26,4 \\
\hline ET -Estima & 46,0 & 13,7 & 40,3 \\
\hline IC -Inseguridad con el contrato & 30,6 & 19,4 & 50,0 \\
\hline IT -Inseguridad con el trabajo & 33,2 & 29,0 & 37,8 \\
\hline DP -Preocupación por las responsabilidades domésticas & 49,5 & 23,6 & 26,9 \\
\hline
\end{tabular}


Tres subdimensiones sobrepasan el umbral del $50 \%$ de riesgo bajo (CO-"Exigencias cognitivas", ES-“Exigencias sensoriales", CR-“Conflicto de rol"), una subdimensión se encontraba en el umbral (IC-"Inseguridad con el contrato"), y tres subdimensiones se encontraban muy cerca del $50 \%$ (IN-“Influencia", ST-"Sentido del trabajo" y $\mathrm{CU}$-"Exigencias cuantitativas").

Hay subdimensiones que no muestran una tendencia clara hacia los extremos de prevalencia de riesgo alto o bajo: IE-“Integración en la empresa", CL-"Calidad del liderazgo", IT"Inseguridad con el trabajo", ET-"Estima", ST"Sentido del Trabajo".

Al analizar las preguntas por separado, las respuestas tienden a concentrarse en los puntajes bajos en la mayoría de las preguntas (en la Tabla 4 se muestran las 18 preguntas con mayor frecuencia de respuesta en cada escala), salvo en "¿Su trabajo requiere atención constante?" y "¿Siente que los problemas en el hogar donde trabaja son también suyos?".

Tabla 4. Porcentaje de respuestas a 18 preguntas seleccionadas, una por cada subdimensión. (3 y 4 puntos = alto riesgo; 0 y 1 punto = bajo riesgo)

\begin{tabular}{|l|l|c|}
\hline Preguntas & $\begin{array}{l}\text { Respuestas de } \\
\mathbf{3} \text { y } \mathbf{4} \text { puntos } \%\end{array}$ & $\begin{array}{c}\text { Respuestas de } \\
\mathbf{0} \text { y } \mathbf{1} \text { puntos } \%\end{array}$ \\
\hline CU. ¿Puede hacer su trabajo con tranquilidad y tenerlo al día? & $-0,30^{* *}$ & 70,5 \\
\hline CO. En su trabajo, ¿tiene usted que tomar decisiones difíciles? & $-0,07$ & 65,5 \\
\hline EM. En general, ¿considera usted que su trabajo le produce desgaste emocional? & $-0,38^{* *}$ & 41,8 \\
\hline EE. En su trabajo, ¿tiene usted que guardar sus emociones y no expresarlas? & $-0,25^{* *}$ & 38,0 \\
\hline ES. ¿Su trabajo requiere atención constante? & $-0,01$ & 19,8 \\
\hline IN. ¿Tiene poder para decidir sobre el ritmo al que trabaja? & $-0,25^{* *}$ & 66,4 \\
\hline CT. ¿Puede decidir cuándo hacer un descanso? & $-0,19^{* *}$ & 61,2 \\
\hline PD. ¿Tiene que hacer lo mismo una y otra vez en forma repetida? & $-0,12$ & 40,0 \\
\hline ST. Las tareas que hace, ¿le parecen importantes? & $-0,18^{* *}$ & 82,9 \\
\hline IE. ¿Siente que los problemas en el hogar donde trabaja son también suyos? & $-0,20^{* *}$ & 27,0 \\
\hline RL. ¿Sabe exactamente qué tareas son de su responsabilidad? & $-0,26^{* *}$ & 90,2 \\
\hline CR. ¿ iTiene que hacer tareas que usted cree que deberían hacerse de otra manera? & $-0,26^{* *}$ & 63,6 \\
\hline CL. ¿Recibe ayuda y apoyo de su inmediato o inmediata superior? & $-0,18^{* *}$ & 50,6 \\
\hline RS. jefes inmediatos, ¿resuelven bien los conflictos? & $-0,21^{* *}$ & 53,1 \\
\hline ET. Mis superiores me dan el reconocimiento que merezco & $-0,29^{* *}$ & 59,9 \\
\hline IC. ¿Está preocupado/a por si la despiden o no le renuevan el contrato? & $-0,27^{* *}$ & 77,2 \\
\hline IT. ¿Está preocupado/a por si la cambian de tareas contra su voluntad? & $-0,21$ & 66,2 \\
\hline DP. Cuando está en el trabajo, ¿piensa en las exigencias domésticas y familiares? & $-0,06$ & 35,3 \\
\hline
\end{tabular}

No identificamos alguna asociación entre escolaridad y subdimensiones de riesgo, ni entre la modalidad de trabajo (puertas adentro / puertas afuera) y riesgo, ni entre riesgo psicosocial y riesgo doméstico.

Tal como para los puntajes de salud, buscamos una correlación entre horas trabajadas y riesgo psicosocial (Spearman), y encontramos correlaciones inversas significativas entre horas de trabajo e influencia, control del tiempo, calidad del liderazgo, relación con superiores y estima, aunque en todos estos casos la correlación fue relativamente pequeña (rho < $-0,20)$.

\section{Resultados de la exploración cualitativa}

Para las dirigentes no constituyó una sorpresa el alto nivel de decisión y de control del tiempo de trabajo que poseen: "Si uno tiene claro lo que tiene que hacer, el ritmo y el orden en que hago las cosas lo decido yo. No hay que 
correr". Saben también que las familias se apoyan completamente en ellas para la gestión del hogar: "Somos importantes en la sociedad para las familias, pensando que ellas nos dejan a cargo de su hogar y al cuidado de sus hijos. Somos un aporte con nuestro trabajo".

La "baja autoestima" sería para ellas uno de los factores que más influyen en la baja salud mental, y no tanto las condiciones de trabajo que mide el cuestionario. Por ejemplo: "Yo les digo [a las otras TCP] que tienen la autoestima tan baja, que no se hace[n] valer."

Preguntamos cuál sería la causa de esta baja autoestima. Ellas refieren que "la mayoría [de las TCP] somos personas de bajos recursos que no vivimos muchas veces en casas bonitas (...) y entramos entonces en este mundo de casas bonitas que no son nuestras casas (...), no queremos estar en el mundo real que nos toca, pero tampoco podemos estar en este otro mundo que es nuestro lugar de trabajo (...) Y ahí empieza este problema de la baja estima".

A esto se agrega el desprecio por la actividad misma de parte los empleadores, que: "Nos dicen que una es una pobre ignorante que no sabe más que lavar los platos y apenas sabe lavarlos. Entonces son un montón de cosas que te van achicando".

Con frecuencia durante la discusión aparece el tema de la vergüenza, la que está asociada con la baja autoestima. Las dirigentes sostienen que deberían hacer "un trabajo" de fortalecimiento de la autoestima de las TCP: "Mientras no nos avergoncemos y [entendamos] que el trabajo doméstico es una profesión o es una labor importante (...) no nos tendríamos que perder".

\section{DISCUSIÓN}

Como se consigna en otros trabajos, ${ }^{8,18}$ las TCP son en su inmensa mayoría mujeres, de mayor edad, y con una escolaridad un poco por sobre la educación básica. Las extranjeras son más jóvenes,con una mayor escolaridad y trabajan mayoritariamente puertas adentro; las chilenas trabajan mayoritariamente puertas afuera.

Los mayores riesgos psicosociales detectados son las exigencias emocionales (EM), lo que parecería confirmar las propuestas de Goldsmith $^{4}$ acerca de que estas trabajadoras sufren un desgaste emocional dada su labor centrada en el cuidado de otros. Sin embargo,al analizar la escala de riesgo doméstico propuesta por las mismas TCP, las tareas centradas en el cuidado (niños menores, ancianos, enfermos, o incluso mascotas, una tarea que parece particularmente ingrata para las TCP) no se asociaron con diferentes niveles de salud ni de riesgo. La subdimensión DP ("Preocupación por las exigencias domésticas"), de obligaciones con la familia propia, tampoco mostró correlación con las escalas de salud. La propuesta de que existe una transferencia de recursos emocionales desde las TCP a los miembros de la familia, ${ }^{7,18}$ si es que existe, no parece tener un impacto sobre el RPSL o la salud de las TCP.

La escala de calidad del liderazgo de las empleadoras tiene un riesgo repartido en los tres niveles, alto, medio y bajo: las empleadoras se comunican bien, se preocupan por el bienestar de las TCP, otorgan ayuda, resuelven bien los conflictos. Por otro lado, la escala de relación con los superiores (RS) muestra un riesgo alto mayor al 50\%, pero al analizar las preguntas de la escala por separado, se observa que la mayoría de las respuestas (alrededor del 50\% en todos los casos) se concentran en niveles bajos, lo que mostraría que reciben un apoyo más o menos constante de sus empleadoras, y que la falta de apoyo es más bien excepcional. Arriagada y Moreno $^{18}$ demostraron que las empleadoras evalúan positivamente el trabajo de sus trabajadoras, considerando a sus empleadas un "apoyo fundamental" para realizar su propio trabajo, lo que coincide con la apreciación que hacen las propias trabajadoras en nuestra experiencia. Pero hay que notar que, aunque la correlación resultó de escasa magnitud (siempre menor a 0,20,rho de Spearman), el exceso de horas de trabajo (hasta 98 horas semanales) está asociado en forma directa a los puntajes de riesgo de liderazgo, aunque no a los puntajes de salud. Las TCP parecen sufrir una situación ambivalente frente a sus empleadoras, porque, por un lado, existe un cierto nivel de liderazgo abusivo, que se expresa en las largas jornadas de trabajo, pero por otro lado las TCP evalúan el liderazgo en un nivel intermedio y no sufren por esto en su salud general ni mental, ni en otro tipo de riesgos psicosociales. 
Es posible que la gran autonomía evidenciada pudiera preservarlas de sufrir otras consecuencias, a pesar de las jornadas agotadoras. La mayoría de ellas puede hacer su trabajo con tranquilidad, lo tienen al día, tienen tiempo suficiente para realizar sus tareas, deciden el ritmo y cantidad de trabajo asignada, y en general su grado de influencia en la tarea es elevado. Arriagada y Moreno ${ }^{18}$, en entrevistas a empleadoras y trabajadoras, muestran que la empleadora delega en la trabajadora labores de gestión, de organización y decisión del trabajo del hogar, y en la mayoría de los casos la transferencia es total. Podemos decir que las TCP son efectivamente las "dueñas de casa".

Por otra parte, las exigencias cognitivas (CO) son también escasas dado que el $66 \%$ de las TCP están en riesgo bajo en esta escala. Esta escala podría interpretarse exactamente a la inversa.El alto riesgo puede encontrarse en realidad en una baja exigencia cognitiva, propia de un trabajo rutinario que no representa desafíos, lo que se confirma con el resultado de la subdimensión posibilidades de desarrollo (PD) en que el 59,9\% de las TCP se encuentran en riesgo alto, y la pregunta sobre trabajo rutinario concentra un $43,0 \%$ de respuestas de alto riesgo. Realizamos un cálculo de correlaciones (Spearman) entre $\mathrm{CO}$ y $\mathrm{PD}$ que resultó negativo (rho $=-0,30$ ) (datos no mostrados), lo que sugiere que una baja exigencia cognitiva se vincula a una escasa posibilidad de desarrollo. En su conjunto, estas escalas muestran que se trata de trabajos rutinarios con un aporte escaso o nulo al desarrollo personal.

La subdimensión ST, sentido del trabajo, muestra un comportamiento polar. Casi el $50 \%$ de las TCP se encuentran en un nivel de bajo riesgo, lo que podría llevar a pensar que su trabajo tiene para ellas mucho sentido. Sin embargo, hay otro 41,4\% que está en riesgo alto. La pregunta que concentró un mayor porcentaje de respuestas en riesgo alto (esto es, "nunca" o "solo unas pocas veces") fue "Las tareas que hace $i$ tienen sentido para usted?", seguida de la pregunta "Las tareas que hace, ¿le parecen importantes?". Por otra parte, la dimensión integración en la empresa (IE), que en este caso es el hogar donde trabajan, muestra una prevalencia de un poco más del 30\% en riesgo alto. Destaca la pregunta
"¿Siente que los problemas en el hogar donde trabaja son también suyos?" con un 56\% de respuestas en riesgo alto, (esto es, "nunca" o "solo unas pocas veces").Tampoco aspiran a quedarse en ese mismo trabajo por el resto de su vida laboral (datos no mostrados). Consideradas en su conjunto, estas subdimensiones parecen mostrar que las TCP se sienten escasamente comprometidas y valoran poco el trabajo y el lugar donde trabajan. Es decir, la presunción de que las TCP transfieren cariño hacia el lugar de trabajo y hacia las personas que cuidan pudiera no tener fundamento. Es posible, incluso, que la distancia afectiva con el hogar donde trabajan pudiera ejercer un rol protector para las TCP, pero también muestra que las TCP no valoran el trabajo que realizan.

En suma, la hipótesis principal de este trabajo no se cumplió. Los hogares donde se desempeñan las TCP no muestran de manera sistemática un alto nivel de riesgo psicosocial en todas las subdimensiones medidas.

Las TCP son asombrosamente autónomas en su trabajo, tienen bajas exigencias cuantitativas, y no parecen afectadas particularmente por una falta de reconocimiento a su trabajo o por tratos inadecuados, valorando en general positivamente la calidad del liderazgo de sus empleadoras. Incluso no parecen preocupadas por la inestabilidad de los contratos (al menos en el caso de las chilenas). Las tareas de cuidado, aun cuando sean múltiples (niños, adultos mayores o enfermos y hasta mascotas), tampoco se relacionan con las exigencias emocionales ni con los niveles de salud mental.

Creemos que el mayor problema que enfrentan estas trabajadoras es la devaluación social y tal vez el desprecio personal hacia su propia actividad. En las conversaciones sostenidas con las TCP, un tema recurrente es la aguda diferencia de niveles sociales entre ellas y la familia a la que sirven, y el contraste entre la disponibilidad de bienes materiales que observan en la casa donde trabajan y sus propios hogares. Las dirigentes entrevistadas sugieren que este es un elemento importante que podría impactar en su estado afectivo y en su autoestima.

En un trabajo cualitativo con entrevistas, Del Campo y Ruiz ${ }^{1}$ demostraron que las TCP recurren a este trabajo a pesar de que tienen conciencia 
de la escasa valoración social que este posee. La minusvaloración podría alcanzar hasta el punto en que muchas TCP ocultan la actividad a la que se dedican ${ }^{1}$. Toledo y Aguilar ${ }^{3}$ sostienen que existe una "delegación del trabajo sucio" desde la empleadora (mujer) hacia la trabajadora (también mujer), permitiendo que la empleadora realice las actividades que son más gratificantes, como su propio trabajo o profesión, o jugar con los niños, y otras actividades similares, delegación que además estabiliza el rol masculino siendo lejano a las tareas domésticas. La contratación de una TCP en consecuencia no está motivada por una necesidad (los hogares de bajos ingresos no contratan TCP a pesar de necesitarlas) ${ }^{19}$, ni por una especialización (no se contrata a una profesional que sabe hacer algo específico que otras personas no saben hacer $)^{19}$, sino que por el rechazo a las tareas que, tanto hombres como mujeres de la casa, se niegan a hacer porque son desagradables, como el aseo de los baños o el planchado, por ejemplo, y eso solo ocurre en hogares de ingresos más altos ${ }^{19}$. En algunos casos, el desprecio de los empleadores por esas tareas es explícito y alcanza niveles de insulto.

Esto hace que la valoración de su trabajo por una TCP sea extremadamente difícil: está haciendo un trabajo "sucio" y que "nadie quiere hacer". Es decir, el trabajo solo se puede sobrellevar mientras no se avergüence.

Es posible concluir que el origen de la mala salud entre las TCP no se origina en un alto RPSL sino en la devaluación social y personal al realizar un trabajo sucio y despreciado y estas dimensiones no parecen ser recogidas por el cuestionario.

\section{REFERENCIAS BIBLIOGRÁFICAS}

1. Del Campo A, Ruiz S. Empoderamiento de Trabajadoras de Casa Particular Sindicalizadas. Psykhe. 2013;22(1):15-28. DOI: 10.7764/psykhe.22.1.635

2. Valenzuela ME, Mora C. Introducción. En: Valenzuela ME, Mora C, editores. Trabajo doméstico: un largo camino hacia el trabajo decente. Ginebra: Oficina Internacional del Trabajo; 2009. p.11-19.

3. Toledo M, Aguilar M. Entre el afecto y las disputas: la casa como espacio laboral feminizado. Espacialidades. 2016;6(1):192219.

4. Goldsmith M. Disputando fronteras: la movilización de las trabajadoras del hogar en América Latina. Amérique Latine Histoire et Mémoire. Les Cahiers ALHIM [en línea] 2007[consultado el 05 dic 2016];14. Disponible en: http://alhim.revues.org/2202

5. Stefoni C. Migración, género y servicio doméstico: mujeres peruanas en Chile. En: Valenzuela ME, Mora C, editor. Trabajo doméstico: un largo camino hacia el trabajo decente. Ginebra: Oficina Internacional del Trabajo; 2009. p.191-232.
6. Stefoni C, editor. Mujeres inmigrantes en Chile. ¿Mano de obra o trabajadoras con derechos? Santiago: Ed. Universidad Alberto Hurtado; 2011.

7. Ehrenreich B, Hochschild AR. Introduction. En: Ehrenreich B, Hochschild AR, editores. Global Woman: Nannies, Maids, and Sex Workers in the New Economy. New York: Henry Holt; 2003. p.4-5.

8. Tijoux ME. Peruanas inmigrantes en Santiago: un arte cotidiano de la lucha por la vida. Polis. 2007;18:1-8.

9. Chile. Ministerio de Desarrollo Social. Encuesta CASEN 2015. Inmigrantes [en línea]; 2016 [consultado el 19 nov 2017]. Disponible en: http://observatorio. ministeriodesarrollosocial.gob.cl/ casen-multidimensional/casen/docs/ CASEN_2015_INMIGRANTES_21122016_ EXTENDIDA_publicada.pdf

10. WHO. Authored by Leka S, Jain A. Health Impacts of Psychosocial Hazards at Work: An Overview. Geneva: World Health Organization; 2010.

11. ILO. Psychosocial factors at work: Recognition and control. (Vol. 56). Report of the Joint ILO/WHO Committee on 
Occupational Health.Geneva: International Labour Office; 1986.

12. Pérez-Franco J. Distribución del riesgo psicosocial laboral en Chile. Rev Chil Salud Pública. 2014;18(1):52-60.

13. Alvarado R, Pérez-Franco J, Saavedra N, Fuentealba C, Alarcón A, Marchetti N, Aranda W. Validación de un cuestionario para evaluar riesgos psicosociales en el ambiente laboral en Chile. Rev Med Chile. 2012;140:1154-1163.

14. Kristensen TS, Hannerz H, Høgh A, Borg V. The Copenhagen Psychosocial Questionnaire - a tool for the assessment and improvement of the psychosocial work environment. Scand I Work Environm Health. 2005;31(6):438-449.

15. Candia M, Pérez-Franco J, González D. Redactado. Manual del método del Cuestionario SUSESO/ISTAS21. Santiago: Superintendencia de Seguridad Social; 2016.

16. Actualización del Protocolo de Vigilancia de Riesgo Psicosocial en el Trabajo. Resolución
Exenta $N^{\circ} 1433$ de 10/nov/2017. Chile. Mnisterio de Salud.

17. Olivares-Tirado P. Perfil del estado de salud de beneficiarios de ISAPREs: Informe preliminar. Documento de Trabajo. Marzo 2005. Superintendencia de ISAPREs. Santiago: Ministerio de Salud; 2005. Disponible en:http://www.supersalud.gob. cl/difusion/665/articles-1068_recurso_1.pdf

18. Arriagada I, Moreno M. La constitución de cadenas globales de cuidado y las condiciones laborales de las trabajadoras peruanas en Chile. En: Stefoni C, editor. Mujeres inmigrantes en Chile. ¿Mano de obra o trabajadoras con derechos? Santiago: Ed. Universidad Alberto Hurtado; 2011. p. 151-191.

19. Devetter F-X. ¿Por qué externalizar las tareas domésticas? Análisis de las lógicas desigualitarias que estructuran la demanda en Francia. Revista de Estudios Sociales. 2013;45 (enero-abril) (Bogotá): 80-95. 